\title{
Reflets
}

Revue d'intervention sociale et communautaire

\section{ST-AMAND, Nérée (2011). Repenser le service social ?, Ottawa, Merriam Print, 324 p.}

\section{Jean-Luc Pinard}

Volume 18, numéro 1, printemps 2012

URI : https://id.erudit.org/iderudit/1012341ar

DOI : https://doi.org/10.7202/1012341ar

Aller au sommaire du numéro

\section{Éditeur(s)}

Reflets, Revue d'intervention sociale et communautaire

ISSN

1203-4576 (imprimé)

1712-8498 (numérique)

Découvrir la revue

Citer ce compte rendu

Pinard, J.-L. (2012). Compte rendu de [ST-AMAND, Nérée (2011). Repenser le service social ?, Ottawa, Merriam Print, 324 p.] Reflets, 18(1), 216-222.

https://doi.org/10.7202/1012341ar d'utilisation que vous pouvez consulter en ligne.

https://apropos.erudit.org/fr/usagers/politique-dutilisation/ 


\section{Repenser le $\backslash \mathrm{ses}^{\mathrm{s}} / \mathrm{c} / \mathrm{s} / \mathrm{s}$ sial?}

ST-AMAND, Nérée (2011). Ottawa, Merriam Print, 324 p.

\section{par Jean-Luc Pinard}

Étudiant au doctorat en service social, Université d'Ottawa

Maîtrise en service social, Université d'Ottawa

Ouvrage de convictions, de réflexions et d'actions, Repenser le social de Nérée St-Amand est une incursion dans le monde des forces personnelles et collectives des individus, lesquelles constituent le principal moteur de changement dans la résolution des problèmes sociaux.

L'œuvre aurait bien pu avoir pour sous-titre Penser autrement pour faire autrement, car c'est là le thème central qui y est développé. Le mot "service " s'insère dans le titre original, Repenser le social, comme une intrusion lexicale dans un champ balayé par un grand angle, celui du social. Contre une vision désespérante de l'humanité,l'auteur appose des exemples de pratiques exemplaires tirés de la riche histoire de personnes qui à travers le monde proposent des valeurs transcendantes de nature à mobiliser l'humanité.

Le premier chapitre de l'ouvrage affirme donc la nécessité de l'engagement dans les dimensions sociale, politique, humanitaire et spirituelle. Écrire sur l'intervention sociale, c'est constater les horreurs et les contradictions de notre société, puis proposer les possibles d'une vision plus inspirante. Ce sont ces possibles que l'on redécouvre au fil des pages.

St-Amand situe le contexte dans lequel évoluent le service social et les professionnels de la relation d'aide. Devant une sombre humanité qu'il décrit à grands traits, l'auteur pose la question : dans nos sociétés industrielles de masse, comment réagir contre 
l'individualisme ambiant et la détérioration des solidarités sociales? En réponse, St-Amand propose une humanité empreinte de noblesse dans laquelle les personnes se centrent sur le "vrai » progrès pour répondre à leurs besoins et s'insurgent contre une autorité qui exige la soumission. L'auteur dresse un bilan de nos accomplissements en matière d'intervention et de nos modes de pensée fortement imprégnés de valeurs marchandes et d'intérêts individualistes. Il en déduit un constat d'échec. En s'éloignant des valeurs fondées sur le pardon, la coopération et le silence, notre société est incapable de résoudre les problèmes complexes des personnes qui souffrent.

Sur quelles assises le travail social a-t-il érigé ses pratiques d'intervention? St-Amand rappelle la vie et l'œuvre de Jane Addams et de Mary Richmond, qui ont marqué d'une manière asymétrique le contenu traditionnel des formations des travailleurs sociaux. Grâce à cet éclairage historique, la dualité et la possible contradiction entre les pratiques cliniques en intervention individuelle prennent leur sens, de même que celles fondées sur l'inclusion, le partage, l'entraide et la solidarité. Dans le deuxième chapitre, l'auteur met en parallèle les écoles de pensée de ces deux pionnières concernant le service social, en faisant ressortir la vision sociopolitique de l'une en opposition au caractère psychosocial et thérapeutique que l'autre préconise. Puis, toujours à la lumière de ces différences fondamentales, St-Amand jette un regard sur les pratiques actuelles, issues d'un système de croyances qui se trouve au cœur des choix qu'auront à effectuer les futurs travailleurs et travailleuses du social : un ardent dévouement dans l'aide caritative ou un engagement résolu pour une meilleure justice distributive? Voilà deux courants idéologiques exposés ici avec brio, dans l'optique d'amener les lecteurs à prendre parti quant aux fondements du service social qui correspondent à leurs convictions.

Parlant de service, l'auteur fait ensuite un retour sur sa carrière d'intervenant pour remettre en question le processus de rédaction des histoires sociales. Outil par excellence d'interprétation des tares, des faiblesses, des carences et des pathologies? À l'aide d'exemples éloquents, St-Amand introduit l'idée d'une approche narrative comme un moyen puissant de reprendre sa vie en mains. L'histoire 
sociale élaborée à partir de l'expertise personnelle via la narration devient l'objet d'une série de démarches d'appropriation. En contexte d'interaction avec un professionnel, « les sujets s'emparent de l'entretien " et peuvent s'engager d'une manière constructive dans un processus de conscientisation et de guérison.

Ce qui rend la lecture du troisième chapitre intéressante et inspirante, ce sont les témoignages de personnes durement éprouvées qui tentent de s'en sortir, les extraits de contes et de récits qui parsèment aussi tout le volume, puis les nombreuses citations de personnages célèbres qui nous sortent des sentiers battus, à l'exemple de celle de Jacques T. Godbout qui exhorte à "créer des liens plutôt que des biens ". Ainsi, alors que les professionnels semblent écrire l'histoire de vie des personnes selon un "deficit-based assessment", les personnes qui écrivent leur propre histoire le font à partir d'un "strenght-based assessment ", une forme de prise de pouvoir et une façon pour soi d'apaiser les souffrances afin de se reconstruire. L'auteur fait état d'une foule d'expériences en matière de pratiques alternatives, aussi intéressantes les unes que les autres, comme le démontre l'utilisation du processus d'écriture et de narration dont les bienfaits thérapeutiques sont incontestables. En ce sens, il rejoint certains principes à l'origine de l'idée du rétablissement, qui n'est pas spécifiquement traité dans l'ouvrage et dont il serait souhaitable d'en examiner les espoirs et les risques de dérive.

Dans le quatrième chapitre, l'auteur pose un regard rétrospectif sur ses années de pratique en travail social. À l'aide de plusieurs exemples, il amène le lecteur à réfléchir sur la formation des travailleurs sociaux. St-Amand fait preuve d'ouverture face à l'univers de l'intervention et aux rapports interpersonnels. S'inspirant de ses nombreux voyages à l'étranger et de son appréciation des diverses cultures, dont celles des peuples autochtones, il aborde en toute confiance des sujets délicats. Sur quels savoirs devrait-on miser? Les discours des ordres professionnels soulignent l'importance de travailler pour le respect des droits et pour la justice sociale. Ils en font une priorité. La formation universitaire prépare-t-elle l'étudiant en conséquence? Les pratiques d'empowerment apprises correspondent-elles à une 
réalité bien ancrée dans les milieux de pratique? À ce chapitre sur les interventions opprimantes, on souhaiterait l'ajout d'une section plus élaborée sur le pouvoir d'agir, une notion incontournable à la base des interventions conscientisantes.

Le cinquième chapitre propose sans ambigüité de ne pas se laisser désigner et nommer par l'usage d'un étiquetage social. St-Amand tente de remettre en questions les épellations traditionnelles — par exemple, « client » ou "cliente » — qui sont stigmatisantes et porteuses d'un pouvoir excessif. Des personnes intériorisent ces vocables souvent dérogatoires. Dans certains cas, l'acceptation d'un diagnostic en tant que partie intégrante de son identité personnelle est présentée par l'intervenant comme une condition essentielle pour l'efficacité supposée du traitement. Qui s'y oppose est présumé vivre dans le déni de son état et refuser honteusement l'aide bienveillante qui lui est offerte. L'auteur remet en question ces mythes lourds de préjugés et porteurs de préjudices potentiellement irrémédiables. Penser autrement, c'est accueillir les personnes comme des sujets de droit, c'est repenser les modèles et les façons de voir la personne, c'est se prémunir contre les attitudes d'arrogance et de mépris liées au regard hautain et distant du professionnel (p.143). Ce chapitre sur «des noms qui en disent long » se termine par la revue de cinq dimensions à retenir dans la façon qu'a le professionnel du service social de nommer ceux et celles qu'il désigne couramment comme ses clients. L'auteur fait le pari qu'il est possible de nommer sans marginaliser.

Dans le sixième chapitre, St-Amand aborde la délicate question de l'adoption, une pratique qui confirme le manque dramatique de soutien du service social à des parents et des familles en difficultés. Il va jusqu'à remettre en question l'existence même de la pratique de l'adoption dans une société où l'imagination fait défaut pour bien accueillir les enfants et les familles dans le besoin alors que s'accentue dans la société l'écart entre les riches et les pauvres. Sortir un enfant de son milieu, de son village ou de son pays est un geste lourd de conséquences. Pour le bénéfice de qui? Y allant d'un point de vue critique, St-Amand met de l'avant une série de principes directeurs en faveur d'une adoption dite de proximité, lorsqu'il n'y a aucune possibilité de laisser l'enfant évoluer dans 
son milieu familial. Rappelant combien cette pratique comporte de souffrances pour l'enfant lui-même, ce chapitre réactive le débat sur l'adoption et sur l'examen d'alternatives possibles à ce que l'auteur appelle un regrettable " exode des berceaux ".

Les trois derniers chapitres de l'ouvrage abordent d'autres particularités de l'intervention sociale. Entre autres, l'auteur déplore que la dimension spirituelle ait été évacuée dans l'intervention sociale. Est-il possible d'appliquer des valeurs liées à la transcendance, à la recherche de sens et à une vision holistique de l'être humain? St-Amand présente cinq grands principes à promouvoir pour un service social à caractère spirituel. Inspirés de grands maitres à penser, ces principes visent des valeurs de noblesse d'âme et un dépassement de soi souvent ignorés des thérapies traditionnelles. Pour y arriver, l'intervenant est invité à reconnaître les dommages de l'oppression chez la personne ou la communauté qui en souffre, à avoir du respect pour l'émotion, sans oublier de compter sur les bienfaits de la méditation et du silence comme pratiques de purification mentale. En somme, il s'agit d'une démarche plus authentique et allant plus loin que le fait d'ajouter un zeste de propos spirituels aux recettes thérapeutiques traditionnelles s'appuyant sur une science psychologisante à courte vue.

Cette recherche de sens aurait-elle aussi sa place en présence d'une personne qui entend des voix? Est-ce que le praticien du social n'a d'autres choix que de s'aligner sur une explication biopsychiatrique du phénomène, la répression du symptôme et la reconnaissance de la maladie? Après une intéressante revue de diverses explications liées aux voix, l'auteur propose quelques pratiques exemplaires, des alternatives qui invitent à penser et à agir autrement. Plus de questions que de réponses émergent de la lecture de ces chapitres. Peut-être y aurait-il lieu d'approfondir les connaissances liées au rôle du travail social dans le vaste domaine de ce qui est couvert par la psychologie, notamment celui des troubles de la pensée, alors qu'un comportement dérangeant ou une souffrance émotionnelle vive adopte vite le nom de la maladie mentale lorsqu'est franchie la porte de l'hôpital, échappant irrémédiablement à tout autre mode d'explications. Quelles conséquences pour des pratiques sociales aidantes? 
St-Amand présente et illustre deux types de pratiques thérapeutiques caractérisant les réseaux actuels de soins, celles liées aux professions exclusives et celles comportant des pratiques inclusives. Les premières s'appuient sur des savoirs experts affirmant connaitre la raison du mal, les moyens de le combattre et de le contrôler, on pourrait dire, à la limite, comme le chirurgien qui procéderait avec succès à l'ablation d'un organe malade. Les secondes, centrées davantage sur l'être humain dans son ensemble, tiennent compte de l'expérience subjective, des savoirs ancestraux, des réseaux sociaux, le tout intégré dans une optique de transformation.

Pas de services sociaux sans " clients ", sans chefs de service, sans l'immense toile d'araignée en forme de rectangles que l'on appelle un organigramme? Pas si sûr. La pratique sociale n'est pas une entreprise de services dont on se doit de cautionner la croissance en s'appuyant sur la docilité d'une clientèle parfois captive, toujours loyale et fidèle. D'une manière insidieuse, la primauté du soin et du rapport dominant des professionnels a tendance à saper les fondements d'une société de droit. Ce que St-Amand appelle les "mouvements de clientèle " arrive difficilement à faire entendre une voix crédible et une parole porteuse de changements au sein des grands réseaux institutionnels.

L'évolution des interfaces entre l'institution, le milieu communautaire et les maisons d'enseignement met en lumière les ambigüités et les contradictions que provoque la coexistence de modèles différents dans l'explication de la souffrance, ses liens, ses causalités et ses manifestations. L'auteur rappelle l'importance pour les milieux de formation de se recentrer sur la richesse des pratiques alternatives et d'aborder autrement la relation d'aide. Il identifie à l'aide de tableaux explicatifs, des modèles de livraison de services et des lieux typiques propices à leur foisonnement. «Dans la foulée des approches médicales et psychologiques, peu d'écoles remettent en question les fondements de la relation entre problèmes humains, ressources professionnelles et clientèle. » (p. 251)

Le lecteur est confronté au dilemme qui déchire les intervenants sociaux qui sont amenés à opter soit pour les pratiques professionnelles institutionnalisées dont le but est de soigner tout 
en exerçant un contrôle social ou les approches de conscientisation et d'éducation populaire qui conduisent à une véritable transformation personnelle et collective. Ainsi, le dernier chapitre du livre constitue un plaidoyer bien appuyé sur la nature, la pertinence et certains modes d'application de pratiques alternatives en travail social, suggérant que le social peut être repensé hors des normes traditionnelles du service, c'est-à-dire ailleurs et autrement que dans des dispositifs institutionnels.

En conclusion, St-Amand rappelle l'esprit de cet ouvrage original qui est de " remettre en question les relations qui nous conferent un pouvoir démesuré ". Après un survol des diverses faiblesses de nos modes de pratique actuels et puisant dans sa propre expérience, l'auteur met en valeur l'expérience personnelle comme moyen d'apprentissage, affirmant qu'elle est beaucoup plus positive que l'aide dispensée par les professionnels, que les gens reçoivent souvent de façon passive et désengagée. Le travail social est un métier d'engagement, le prolongement naturel d'une posture de citoyen éclairé. Sa vision doit donc tendre vers une plus grande conscientisation. St-Amand énonce sans ambages que les travailleurs sociaux doivent trouver des pratiques qui leur permettent de promouvoir la justice sociale et la participation des gens qu'ils desservent. Il pense qu'ils peuvent changer le système actuel en traitant des causes structurelles qui gardent les gens dans la pauvreté économique et sociale.

Repenser le social s'adresse à ceux et à celles qui aspirent à devenir praticiens du social, qui s'engagent dans la promotion et la construction d'une société fondée sur la dignité humaine ainsi qu'à toute personne qui désire enrichir et mettre à jour sa vision du social, incluant les étudiants désireux de faire par leur profession une heureuse différence dans la vie des gens. L'ouvrage s'adresse au cœur et à l'imagination. Il s'adresse aux artisans de l'esprit et à ceux et à celles qui, indignés et persévérants, continuent à lutter pour une société meilleure et plus égalitaire.

\section{Note}

Merci à Claude Snow, de Caraquet, Nouveau-Brunswick, pour son aide dans la rédaction de ce texte. 